\title{
Can diffusion and T2 star-weighted magnetic resonance imaging aid in the diagnosis of ectopic endometrium?
}

\author{
Mariam Raafat ${ }^{1,2^{*}}$ (D), Soha H. Talaat ${ }^{2}$, Salma M. Abdelghaffar ${ }^{2}$ and Engy A. Ali ${ }^{2}$
}

\begin{abstract}
Background: Endometriosis is a common gynecologic disorder characterized by the implantation of the endometrial tissue ectopically outside the endometrial cavity. It affects about $10 \%$ of females at the childbearing period and is estimated to be present up to $20-50 \%$ in women complaining of infertility. While laparoscopy is considered the mainstay for diagnosis, magnetic resonance imaging (MRI) is recognized as a useful tool for definitive diagnosis, pre-surgical planning, and determining whether the patient will require multi-specialty involvement. The aim of this study is to evaluate the performance of MRI with the addition of diffusion-weighted imaging (DWI) and T2 star (T2*) to conventional MRI, for the accurate assessment of ectopic endometrium.

Results: Endometriotic lesions that showed diffusion restriction on DWI were $80.7 \%$, and $96.1 \%$ of the endometriotic lesions had signal voids on the T2*W sequence, whereas only $65.4 \%$ of the lesions had typical signal intensities on T1WI and T2WI. Diagnostic performance of the MRI examination was improved by the use of the diffusion sequence and better improved by the $\mathrm{T}^{*}$ sequence, compared to the conventional MR protocol sensitivity (SE) $=96.12 \%$ and specificity $(\mathrm{SP})=85.7 \%$ in T2*-weighted images, $\mathrm{SE}=80.7 \%$ and $\mathrm{SP}=71.4 \%$ in DWI, and $\mathrm{SE}=65.4 \%$ and $\mathrm{SP}=71.4 \%$ in conventional MRI. P value for conventional MRI was 0.1 , which is of no statistical significance $(p<0.05)$. P value for DWI was 0.016 , which is statistically significant $(p<0.05)$. P value for T2*WI was 0.001 , which is more statistically significant $(p<0.05)$ and could be adequately correlated with laparoscopy.

Conclusion: DWI and T2* significantly increase MRI diagnostic accuracy by allowing the detection of the hemorrhagic character of the endometriotic lesions. Studies with a large sample size are needed to confirm that they can replace invasive laparoscopy for the diagnosis of endometriosis.
\end{abstract}

Keywords: Endometriosis, MRI, DWI, T2*WI

\section{Background}

Endometriosis is a common gynecologic disorder characterized by the implantation of endometrial tissue ectopically outside the endometrial cavity. The prevalence is approximately $10 \%$ of females at the childbearing period and is estimated to be present up to $20-50 \%$ in women complaining of infertility [1]. It is known to cause various

\footnotetext{
* Correspondence: mariam_raafat@yahoo.com

'Diagnostic and Intervention Radiology Department, Cairo University Hospitals, Kasr Al-Ainy, El-Manial, Cairo 11956, Egypt

${ }^{2}$ Diagnostic and Interventional Radiology Department (Women's Imaging Unit), Kasr ElAiny Hospital, Cairo University, Giza, Egypt
}

symptoms, including chronic pelvic pain, dysmenorrhea, dyspareunia, and infertility [2]. Ultrasound (US) is usually the first modality used in the diagnosis of endometriotic lesions; however, MRI has many advantages to US with more reproducible image acquisition over a larger field of view allowing the detection of disease outside the pelvis. Information collected from multiple sequences leads to better characterization of ovarian lesions [3].

While laparoscopy is considered the mainstay for diagnosis, MRI is recognized as a useful tool for definitive diagnosis, pre-surgical planning, and determining whether the patient will require multi-specialty involvement [4].

\section{Springer Open}

(c) The Author(s). 2021 Open Access This article is licensed under a Creative Commons Attribution 4.0 International License, which permits use, sharing, adaptation, distribution and reproduction in any medium or format, as long as you give appropriate credit to the original author(s) and the source, provide a link to the Creative Commons licence, and indicate if changes were made. The images or other third party material in this article are included in the article's Creative Commons licence, unless indicated otherwise in a credit line to the material. If material is not included in the article's Creative Commons licence and your intended use is not permitted by statutory regulation or exceeds the permitted use, you will need to obtain permission directly from the copyright holder. To view a copy of this licence, visit http://creativecommons.org/licenses/by/4.0/. 
Though there is a wide range of imaging appearances of endometriosis, yet their hemorrhagic elements are its main characterizing imaging feature aiding in its diagnosis [5].

Endometriomas contain various stages of hemorrhage, thus produces a wide range of signal behaviors; the shading sign can vary from faint shading to complete signal voiding. Therefore, it is assumed that the degree or volume of shading would differ between endometriomas and hemorrhagic cysts [6].

Diffusion-weighted imaging (DWI) contributes structural and functional information about the biological tissues, without using ionizing radiation or intravenous contrast. Endometriotic implants may show diffusion restriction [7]. A role for DWI is distinguished in the differentiation between endometriomas and hemorrhagic cysts with significantly lower apparent diffusion coefficient (ADC) values in endometriomas when compared to hemorrhagic ovarian cysts at all $b$ values [8].

A characteristic feature of endometriosis is cyclic hemorrhage caused by ectopic endometrium resulting in deposition of concentrated hemosiderin-laden within the cyst. Identification of this hemosiderin deposit by MRI helps in the diagnosis of endometriomas [9].

$\mathrm{T} 2$-weighted images show magnetic susceptibility effects as signal voids. It is sensitive to hemosiderin; consequently, it aids in the detection of old hemorrhagic content in endometriotic implants and endometriomas [2].

The purpose of this study is to evaluate the performance of MRI with the addition of diffusion-weighted imaging and $\mathrm{T}^{*}$ to conventional MRI, for the accurate assessment of endometriosis.

\section{Methods}

\section{Patients}

This cross-sectional prospective study was done during the diagnostic step or the pre-operative assessment of endometriosis, between August 2019 and August 2020.

Though MRI was performed on 120 patients, yet our quantitative studies included only the surgically/laparoscopically confirmed lesions which were 60 patients with 66 lesions. Their ages ranged from 17 to 44 years. The mean patient age was $31.4 \pm 8.13$ years.

\section{Inclusion criteria}

The following are the inclusion criteria:

- Cystic adnexal/pelvic masses with typical endometriosis signal in ultrasound

- Infertile females seeking fertility, who were referred from the obstetrics and gynecology department with ultrasound showing suspicion of endometriosis

- Women complaining of chronic pelvic pain, especially if it was cyclic

\section{Exclusion criteria}

The following are the exclusion criteria:

- Patients with complex ovarian masses, those containing solid lesions.

- We also excluded patients who have a contraindication to MRI examination, such as those having aneurysmal clips and pacemakers.

\section{Magnetic resonance imaging}

The MRI was conducted with a 1.5-T magnet device (Gyroscan INTERA and ACHIEVA, Philips Medical Systems, the Netherland), using a pelvic phased-array coil with eight channels.

MRI device: the protocol included T1W, with and without fat saturation sequences; T2W; DWI; and T2*weighted imaging. MRI results were correlated with histopathological findings in patients who underwent laparoscopy, laparotomy, or open surgical treatment.

Imaging was performed regardless of the stage of the menstrual cycle.

\section{Patient preparation}

The following are the patient preparations:

- Fasting (4-6h) before the examination.

- Bowel enema administered approximately $2-3 \mathrm{~h}$ before the examination, with 2 doses of an oral laxative ( $5 \mathrm{mg}$ bisacodyl per dose) the day before imaging.

- Follow a low-residue diet on the day before and the day of the examination.

- Anti-peristaltic agent is administered intravenously before the examination.

- Ten milligrams of butylescopolamine (Buscopan; Boehringer Ingelheim, Ingelheim, Germany).

- Moderate bladder filling, by not urinating $1 \mathrm{~h}$ before the study.

- An overfilled bladder may produce movement artifacts and alter the anatomy of the pelvis.

\section{Patient position}

Patient was imaged in the supine position.

The protocol used in our study is summarized in Table 1.

\section{Image analysis}

MR image interpretation was performed by 2 expert radiologists with more than 10 years of experience in MRI who were blinded about each other's imaging findings, and they were not aware about the pathology.

In our MRI interpretation, following Foti et al. [2], the criteria considered for the prediction of endometriosis were as follows: 
Table $1 \mathrm{MRI}$ protocol performed in the current study

\begin{tabular}{lllllll}
\hline Sequence & TR $(\mathbf{m s})$ & TE $(\mathbf{m s})$ & FOV $(\mathbf{m m})$ & Matrix & Slice thickness $(\mathbf{m m})$ & Flip angle \\
\hline T2 sagittal & 3000 & 90 & $290 \times 290$ & $208 \times 205$ & 4 & 90 \\
T2 axial & 3700 & 100 & $288 \times 350$ & $292 \times 180$ & 5 & 90 \\
T1 axial & 500 & 10 & $260 \times 216$ & $263 \times 171$ & 5 & 90 \\
T1 FS axial & 530 & 8 & $240 \times 240$ & $240 \times 190$ & 5 & 90 \\
T2 coronal & 3000 & 90 & $300 \times 300$ & $272 \times 200$ & 4.5 & 90 \\
DWI (b0, 500, 1000) & 2000 & 77 & $240 \times 240$ & $124 \times 100$ & 5 & 90 \\
T2* & 500 & 4.6 & $240 \times 240$ & $256 \times 256$ & 4 & 20 \\
\hline
\end{tabular}

Slice gap is one $\mathrm{mm}$ and flip angle $90^{\circ}$ in all sequences, except $\mathrm{T} 2^{*}, 20^{\circ}$

Abbreviations: FOV field of view, FS fat suppression, DWI diffusion-weighted imaging

(1) High signal on T1-weighted images with and without fat suppression

(2) Intermediate to low T2WI signal with shading

(3) Restriction at diffusion

(4) Punctate or curvilinear signal voids at T2*WI.

Our data analysis focused upon DWI SI and measured ADC values, as well as $\mathrm{T} 2 *$ signal for the cystic adnexal masses.

\section{Diffusion-weighted images}

DW images were observed for the presence of a persistent high signal (restricted diffusion) with an increasing $b$ value in correlation with the hemorrhagic components of the included lesions. Matched ADC maps were done using a Phillips Advantage windows workstation with functional tool software. We measured the ADC values by manually applying region of interest (ROI) on the restricted portions of the lesions.

ROI was specifically placed at areas of bright SI on DW images. For lesions with a hemorrhagic component, a large ROI was applied to cover as much as possible of the pathology, in case of lesions with multiple small scattered hemorrhages; multiple ROIs were applied to the areas of concern, and an average was obtained.

\section{Surgical findings}

Surgical findings were considered as the gold standard.

The possible pathology of the endometriosis was suggested by conventional MR imaging, diffusion-weighted imaging, and $\mathrm{T} 2 *$-weighted imaging and had been correlated with surgical pathology specimen in patients who have undergone laparoscopy, laparotomy, or open surgical treatment.

\section{Statistical analysis}

We used software (SPSS for Windows, version 10.0.1, 1999; SPSS, Chicago, Ill) for statistical analysis.

Qualitative variables are described as frequencies and percentages. To assess the diagnostic performance, the sensitivity, specificity, positive predictive value, and negative predictive values were estimated from the associated contingency table. Diagnostic performance values of MRI were only evaluated for the patients who underwent laparoscopy, laparotomy, or open surgery.

MRI signal of endometriosis lesions in T1-weighted images with and without fat suppression, T2-weighted images, diffusion-weighted images, and in $\mathrm{T} 2 *$ sequence were compared using percentages, by analyzing the endometriotic lesions found in operated patients.

A $P$ value less than 0.05 was considered statistically significant in all analyses.

\section{Results}

Though the study included about one hundred and twenty cases, yet histopathologic examination was the only confirmatory method for the lesions; thus, our quantitative studies included only the surgically/laparoscopically confirmed lesions. MR image interpretation was performed by 2 expert radiologists with more than 10 years of experience. The inter-observer reliability between the two radiologists was calculated through the Cohn Kappa test which was 0.97, indicating perfect agreement.

Sixty-six pathologically proven lesions in sixty patients were identified as follows:

- Thirty-four ovarian endometriomas

- Eight abdominal wall cesarean scar endometriosis

- Two bladder endometriosis

- Eight adenomyosis

- Fourteen non-endometriotic/hemorrhagic cysts

Surgical/laparoscopic exploration and resection were performed in 60 patients (30 did laparoscopy, 28 had open surgeries, and 2 had undergone cystoscopy) with 66 lesions, diagnosed as follows: 34 endometriomas in 28 patients (6 bilateral (Fig. 1) and 22 unilateral), 8 adenomyosis, 8 abdominal scar endometriosis, 2 bladder endometriosis, and 14 hemorrhagic cysts.

MRI findings of the surgically proven endometrial lesions are shown in Table 2. 

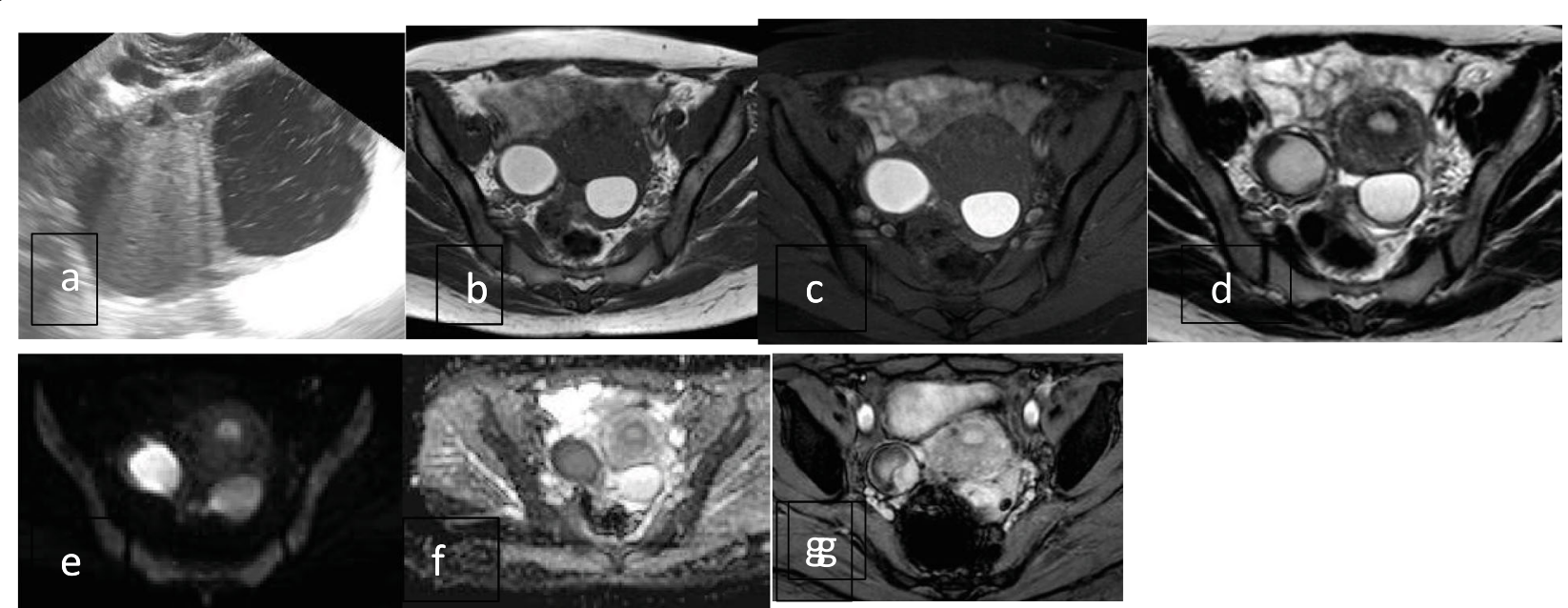

Fig. 1 (a) TV-US showing bilateral ovarian cysts; the right is suggestive of endometrioma (ground glass), and the left is suggestive of hemorrhagic cyst (fishnet appearance). (b) Axial MRI T1Wl, showing bilateral ovarian cysts eliciting bright T1. Not suppressed with fat suppression (c). Showing subtle T2 shading (d). With diffusion restriction (e, f). Right curvilinear and left punctate signal voids in T2* (g). Histopathological diagnosis: bilateral ovarian endometriosis

Thus, we noted that among the surgically confirmed cases of endometriosis, 32 out 52 cases showed typical appearance on conventional MRI, 42 out of 52 cases showed diffusion restriction, and 50 out 52 cases showed signal voids at T2 star-weighted MRI images. MRI analysis with the aid of diffusion sequence showed excellent outcomes with the complete pathology specimen in 42 lesions ( 26 endometriomas, 6 adenomyosis, 8 abdominal scar endometriosis, and 2 bladder wall endometriomas): $\mathrm{SE}=80.7 \%(n=42 / 52)$ true positives and 10 false negatives.

The mean apparent diffusion coefficient (ADC) value of the endometriotic lesions and adenomyosis was 1.03 with a minimum of 0.25 , a maximum of 2.3 , and 0.51 standard deviation. On the other hand, the mean ADC values of functional hemorrhagic cysts were significantly lower $\left(0.71 \pm 0.29 \times 10-3 \mathrm{~mm}^{2} / \mathrm{s}\right)$.

With the addition of $\mathrm{T} 2 *$-weighted sequence, the results improved where 50 out of 52 lesions were true positive (showing signal voids) (32 ovarian endometrioma (Fig. 2), 8 adenomyosis (Fig. 3), 8 CS scar endometriosis (Fig. 4), and 2 bladder wall endometriosis (Fig. 5)): $\mathrm{SE}=96.1 \%$ $(n=50 / 52)$ true positives and 2 false negatives.

We carried out a comparative study for the diagnostic performance of the conventional MRI pelvic examination in addition to each of DWI and T2* in the assessment of the pathologically diagnosed cases (Table 3).

$P$ value is calculated by the Fisher exact test. $P$ value for conventional MRI was 0.1 , which is of no statistical significance $(p<0.05)$. $P$ value of DWI was 0.016 , which is statistically significant $(p<0.05) . P$ value for T2*WI was 0.001 , which is more statistically significant and could be adequately correlated with laparoscopy $(p<0.05)$ (Fig. 6).

\section{Discussion}

Endometriosis is a benign and chronic gynecologic disorder, where there is ectopic implantation of endometrial glands and stroma outside the uterine cavity. It has been shown to cause various symptoms, including chronic pelvic pain, dysmenorrhea, dyspareunia, and/or infertility [2].

Table 2 MRI findings of surgically proven cases

\begin{tabular}{|c|c|c|c|c|c|c|c|}
\hline \multirow[t]{2}{*}{ Site } & \multicolumn{2}{|c|}{$\begin{array}{l}\text { High intensity on } \mathrm{T} 1 \text { weighted } \\
\text { and low/shading on } \mathrm{T} 2\end{array}$} & \multicolumn{2}{|c|}{ Restricted diffusion } & \multicolumn{2}{|c|}{ Signal void on $\mathrm{T} 2^{*}$} & \multirow{2}{*}{$\begin{array}{l}\text { Surgical findings } \\
\text { No. }\end{array}$} \\
\hline & Present & Absent & Present & Absent & Present & Absent & \\
\hline Endometriomas & $16(47.05 \%)$ & $18(52.95 \%)$ & $26(76.47 \%)$ & $8(23.53 \%)$ & $32(94.11 \%)$ & $2(5.89 \%)$ & 34 \\
\hline Abdominal scar & $8(100 \%)$ & $0(0 \%)$ & $8(100 \%)$ & $0(0 \%)$ & $8(100 \%)$ & $0(0 \%)$ & 8 \\
\hline Bladder & $2(100 \%)$ & $0(0 \%)$ & $2(100 \%)$ & $0(0 \%)$ & $2(100 \%)$ & $0(0 \%)$ & 2 \\
\hline Adenomyosis & $6(75 \%)$ & $2(25 \%)$ & $6(75 \%)$ & $2(25 \%)$ & $8(100 \%)$ & $0(0 \%)$ & 8 \\
\hline Non-endometriomas & $4(28.57 \%)$ & $10(71.43 \%)$ & $4(28.57 \%)$ & $10(71.43 \%)$ & $2(14.28 \%)$ & $12(85.72 \%)$ & 14 \\
\hline
\end{tabular}



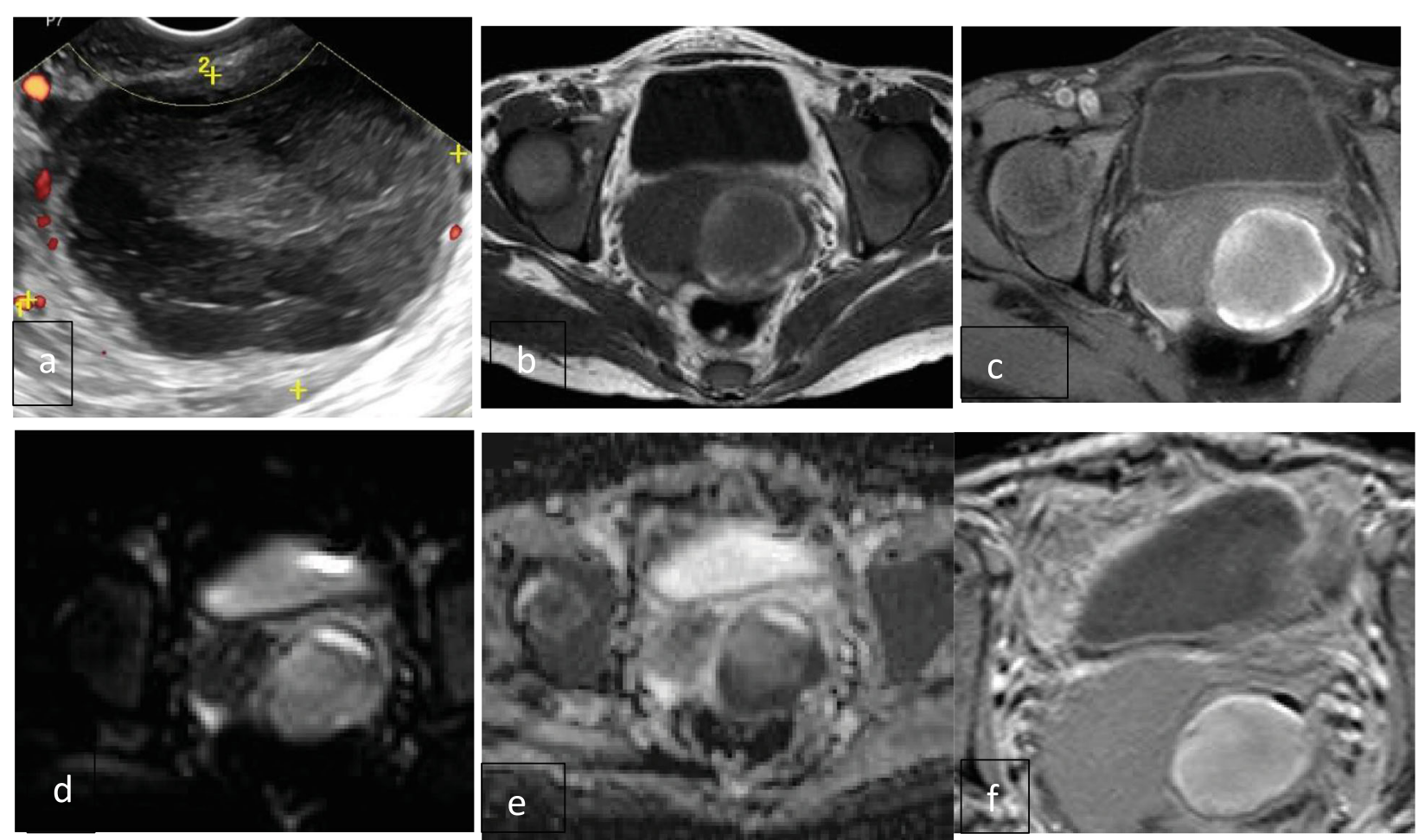

Fig. 2 (a) TV-US showing left ovarian chocolate cyst. It elicits heterogenous signal with peripheral high signal on T1 (b), that is not suppressed on T1 fat suppression (c), and no significant shading (not shown) nor significant diffusion restriction (d, e) however punctate peripheral T2* signal void (f). Histopathological diagnosis: left ovarian endometriosis

Endometriomas can occur unilaterally or bilaterally, and approximately $28 \%$ of endometrioma patients have bilateral endometriomas [10].

In our study, bilaterality was observed in $35 \%$ of endometriomas. However, as mentioned by Lee et al. [6], bilaterality cannot be used to reliably differentiate endometriomas from other cystic lesions.

There is a range of imaging features in endometriosis, but the hemorrhagic character of lesions markedly aids in their characterization [5].

Shading sign in T2-weighted MRI images is typical of endometriomas that helps differentiate it from other hemorrhagic ovarian lesions. Menstrual bleeding over time and long term produce high iron concentration, protein, and methemoglobin with consequent $\mathrm{T} 2$ shortening [11]. Lee et al. [6] reported that the shading sign had a sensitivity and specificity of $90-98 \%$ for the diagnosis of endometriomas; however, his study included more than $80 \%$ of the lesion endometriomas. Outwater and Dunton [12] conducted a study, where the sensitivity and specificity of T2 shading were only $68 \%$ and $83 \%$, respectively. In our study, conventional MRI with high T1-weighted images and shading sign in T2-weighted images exhibited a sensitivity of $65.4 \%$ and a specificity of $71.4 \%$. We believe that the shading is not a reliable
MRI sign for differentiating endometriomas from hemorrhagic cysts.

Blood or hemosiderin within endometriomas shortens $\mathrm{T} 1$, causing a consequent reduction in ADC values; therefore, DWI may distinguish endometriomas from other cystic lesions [13]. In our study, 42 out of 52 pathologically proven endometriotic lesions and 4 non-endometrial hemorrhagic cysts showed restricted diffusion, with SE, SP, PPV, and NPP of $80.7 \%, 71.4 \%, 91.3 \%$, and $50 \%$, respectively. Thus, DWI has shown an increase in sensitivity, PPV, and NPV yet similar specificity, when compared to conventional MRI. This is comparable to Abd ElDayem et al.'s [14] study where 11 out of 14 cases were restricted in DWI representing $78.57 \%$ sensitivity.

However, due to the presence of overlap between the ADC values of endometriomas and hemorrhagic cysts, the diagnosis should be based upon the combination of clinical history and conventional MRI together with DWI findings and should not be based on DWI alone.

In 2002, it was suggested by Moteki et al. [15] that endometriomas had lower ADC values $(0.91 \pm 0.47 \times$ $\left.10^{-3} \mathrm{~mm}^{2} / \mathrm{s}\right)$ than other pelvic cysts $\left(2.82 \pm 0.80 \times 10^{-3}\right.$ $\mathrm{mm}^{2} / \mathrm{s}$ ). Busard et al [16]. reported that ADC values of endometriomas are $1.10 \pm 0.38 \times 10^{-3} \mathrm{~mm}^{2} / \mathrm{s}$ ), and Lee et al. [6]. calculated ADC values for endometriomas to 


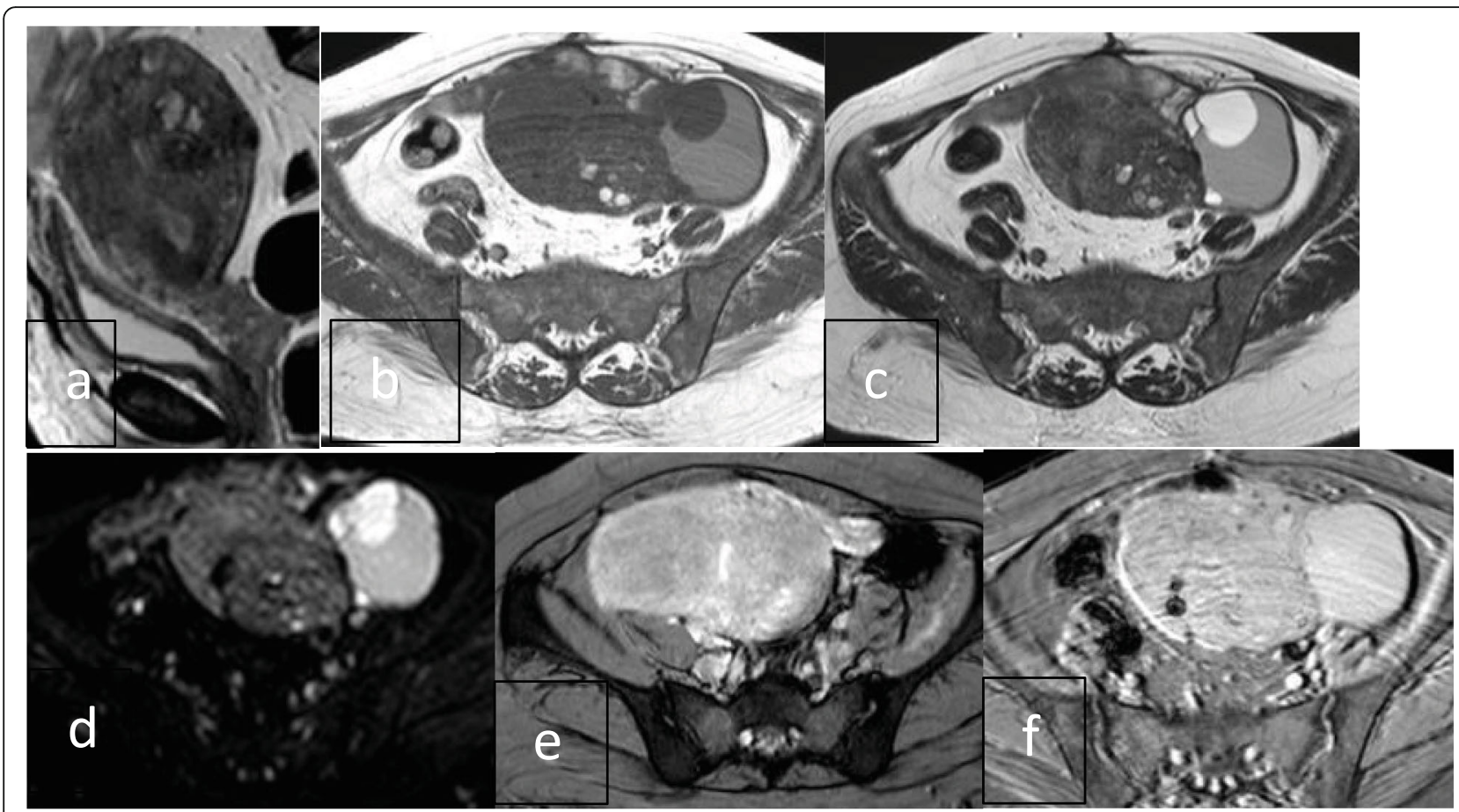

Fig. 3 (a) Sagittal T2 MRI showing diffuse thickening and irregularity of the posterior junctional zone. The posterior fundal myometrium is heterogeneous showing cystic changes bright in $\mathrm{T} 1$ and $\mathrm{T} 2(\mathbf{b}, \mathbf{c})$. With diffusion restriction $(\mathbf{d}, \mathbf{e}) ; \mathrm{T} 2^{*}$ revealed signal void punctate foci (f), suggesting adenomyosis. N.B: left adnexal cystic lesion showing facilitated diffusion. Histopathological diagnosis: focal adenomyosis and left peritoneal inclusion cyst

be $1.06 \pm 0.38 \times 10^{-3} \mathrm{~mm}^{2}$. These studies' results are comparable to our study, which had mean ADC values for endometriomas of $1.03 \pm 0.51 \times 10^{-3} \mathrm{~mm}^{2} / \mathrm{s}$.

Methemoglobin and hemosiderin are especially well visualized on $\mathrm{T} 2 \%$-weighted image that is a MR sequence that is sensitive to blood by-products. The visualization of signal voids is caused by the local heterogenicity of the magnetic field, resulting from hemorrhagic products [5].

Takeuchi et al. [17] reported that $\mathrm{T}^{*}$ improved the characterization of endometrioma by detecting

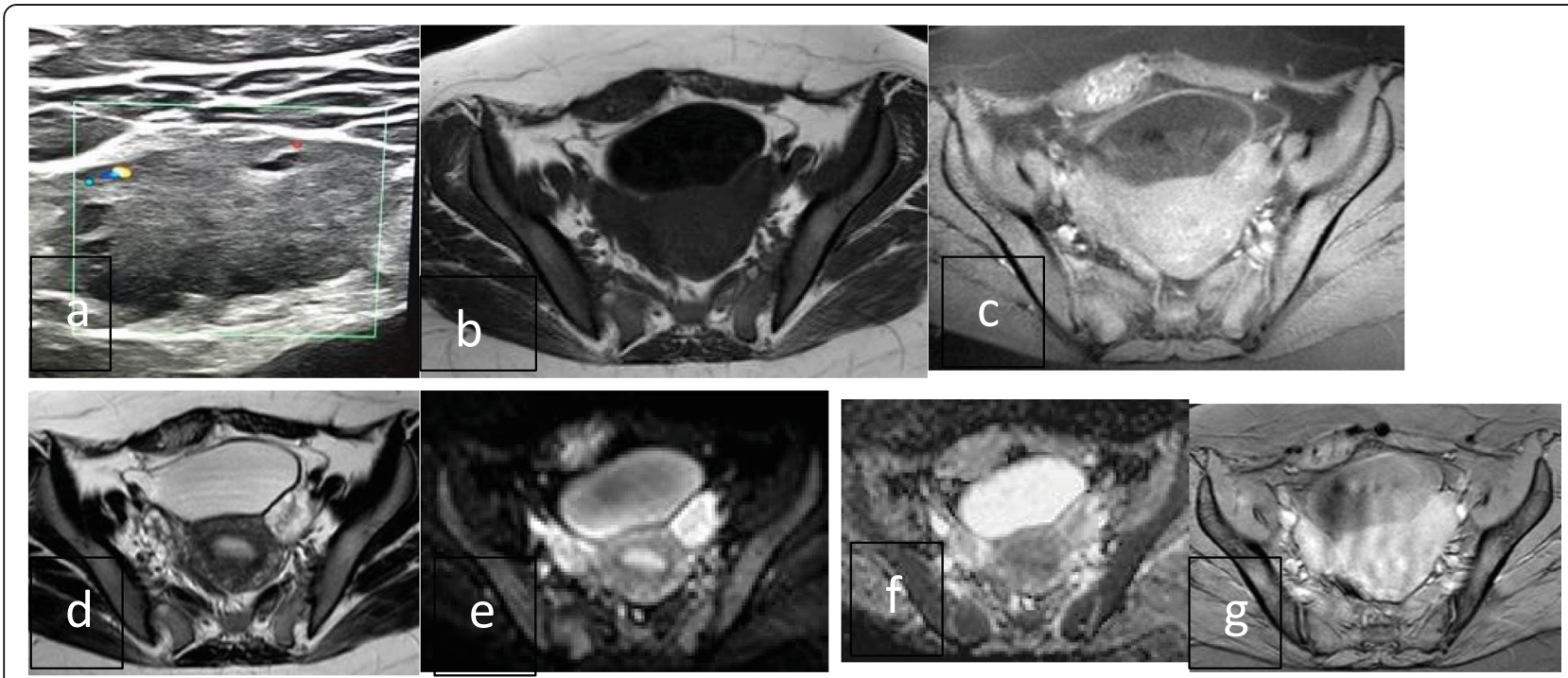

Fig. 4 (a) US of the anterior abdominal wall showing a well-defined infiltrative lesion implicating the right rectus muscle, infiltrating both the anterior and posterior rectus sheaths, and eliciting intermediate T1 SI (b), with high T1FS (c), intermediate T2 SI (d). It shows focal areas of diffusion restriction on DWIs $(\mathbf{e}, \mathbf{f})$, and punctuate signal voids on $\mathrm{T}^{*} \mathrm{WI}(\mathbf{g})$ 


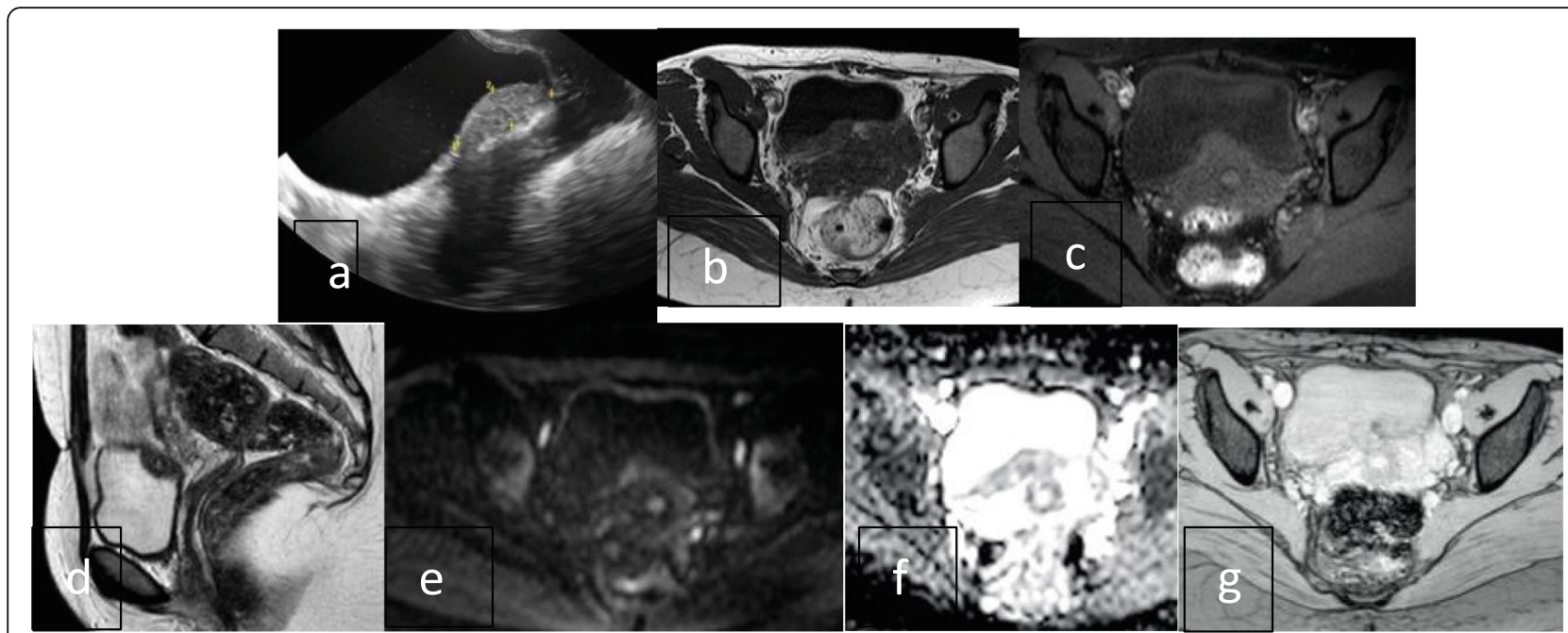

Fig. 5 (a) Transabdominal US showing focal thickening of the dome of the urinary bladder, eliciting intermediate to high T1 (b, c) and intermediate T2 SI (d). It shows diffusion restriction on DWIs (e, $\mathbf{f})$ and signal void on T2* WI $(\mathbf{g})$

signal void along the cyst wall as hemosiderin deposition. Later in 2015, Takeuchi et al. [18] proved that signal voids as hemorrhagic components on $\mathrm{T} 2 *$ were also observed in extra-ovarian endometriosis.

Moreover, Pin et al. [5] suggested that $\mathrm{T}^{*}$ might be useful and added as a sequence for treatment planning of endometriosis. Pin et al .[5] in their study stated that the overall diagnostic performances of MRI with $\mathrm{T} 2 *$ were improved regarding the evaluation of adnexal and deep infiltrating endometriosis with an increase of the sensitivity from 88.2 to $94.1 \%$ and specificity from 68.8 to $73.3 \%$.

In our study, with the addition of T2*WI, 50 out of 52 endometriomas/adenomyosis showed signal voids. Thus, the sensitivity improved from $65.4 \%$ by conventional imaging to $96.15 \%$ with the addition of $\mathrm{T} 2 \%$. Also, the specificity improved from $71.4 \%$ by conventional imaging to $85.7 \%$ with the addition of $\mathrm{T} 2 \%$.

So, in cases with doubtful ultrasound and conventional MRI findings in confirming the diagnosis of endometriosis, DWI and T2* can represent an additional diagnostic tool in order to differentiate endometriomas from other cystic lesions.
Our study had few limitations. Although the study was originally done on 120 cases, yet only 66 lesions were included in the study as we considered the pathology is our gold standard; so, a larger sample size is needed. Susceptibility artifacts caused by intestinal gas may camouflage the detection of signal voids resulting from the presence of blood by-products within especially in cases of ultra-small and deep endometriotic foci. One of our patients was excluded from the study due to susceptibility artifacts caused by colonic overdistension, which limited the visibility of the pelvis. Two lesions were reevaluated by two radiologists to sort out the discrepancies resulting from susceptibility artifacts.

\section{Conclusion}

Endometriosis is the most common cause of chronic pelvic pain in reproductive-age women. It affects approximately $10 \%$ of women at the childbearing period.

It is found in $20-50 \%$ of women with infertility and nearly $90 \%$ of women with chronic pelvic pain. It has a significant impact on the quality of life of women. In addition to the characteristic symptoms of dysmenorrhea,

Table 3 Sensitivity, specificity, positive predictive value, negative predictive values of conventional MRI; DWI and signal void in T2* images

\begin{tabular}{llll}
\hline & Conventional MRI & DWI with ADC maps & Signal voids \\
\hline Sensitivity (\%) & 65.4 & 80.7 & 96.15 \\
Specificity (\%) & 71.4 & 71.4 & 85.7 \\
PPV (\%) & 89.47 & 91.3 & 96.2 \\
NPV (\%) & 35.7 & 50 & 85.7 \\
P value & 0.106 & 0.016 & 0.001 \\
Accuracy (\%) & 66.7 & 78.8 & 93.9 \\
\hline
\end{tabular}

PPV positive predictive value, NPV negative predictive value 


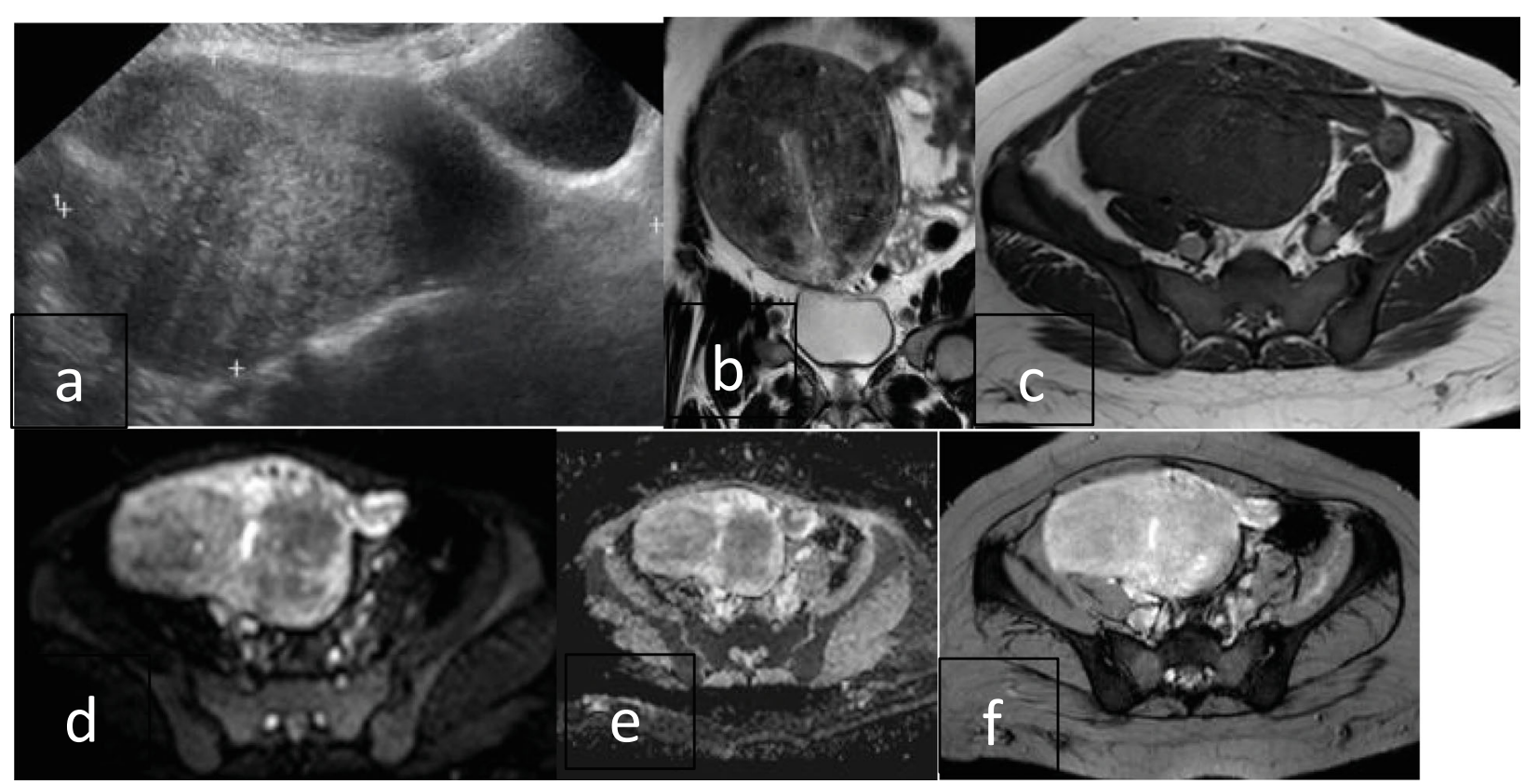

Fig. 6 (a) Transabdominal US showing bulky uterus with sunray appearance. Diffuse adenomyosis, evident by the markedly enlarged uterus, with diffuse marked thickening of the heterogeneously hypointense junctional zone showing scattered tiny cystic changes within (b, $\mathbf{c})$. There is no restricted diffusion on DWI $(\mathbf{d}, \mathbf{e})$. However, the is peripheral curvilinear signal voids on T2*WI (f)

non-cyclic pelvic pain, infertility, and others, it causes psychological, marital, and social distress.

Our study was performed during the diagnostic step or the pre-operative assessment of endometriosis. The MRI was conducted with a 1.5-T MRI device; the protocol included T2W and T1W, with and without fat saturation sequences complemented with a DWI, ADC map, and a T2* WI, and the diagnostic performance values were evaluated. MRI results were correlated with surgical findings in patients who underwent laparoscopy.

Endometriotic lesions had restricted diffusion on DWI in $80.7 \%$, and $96.1 \%$ of the endometriotic lesions had signal losses on the $\mathrm{T} 2 \% \mathrm{~W}$ sequence, whereas only $65.4 \%$ of the lesions had typical signal intensities on T1WI and T2WI. Thus, the diagnostic performance of the MRI examination was improved by the use of the diffusion sequence and better improved by the $\mathrm{T} 2 \%$ sequence, compared to the conventional.

$P$ value for conventional MRI was 0.1 which is not considered statistically significant when associated with laparoscopy ( $\mathrm{p}<0.05)$. P value for DWI was 0.016, which is considered statistically significant when associated with laparoscopy $(\mathrm{p}<0.05)$. $\mathrm{P}$ value for T2*WI was 0.001 , which is considered statistically significant when associated with laparoscopy $(\mathrm{p}<0.05)$.

Diffusion-weighted imagining and $\mathrm{T} 2 *$ significantly increases MRI diagnostic accuracy by allowing the detection of the hemorrhagic character of endometriotic lesions. Studies with a large sample size are needed to confirm that they can replace invasive laparoscopy for the diagnosis of endometriosis.

We recommend the addition of diffusion-weighted imaging and $\mathrm{T} 2 \%$-weighted imaging to all cases of suspected endometriosis.

\section{Abbreviations}

MRI: Magnetic resonance imaging; T2*: T2 star; US: Ultrasound;

DWI: Diffusion-weighted imaging; SE: Sensitivity; SP: Specificity; FOV: Field of view; FS: Fat suppression; ADC: Apparent diffusion coefficient; PPV: Positive predictive value; NPV: Negative predictive value; ROI: Region of interest

\section{Acknowledgements}

No acknowledgments

Authors' contributions

MR contributed to the study conception and design, collection of patients' data, and writing and editing of the manuscript. STH contributed to the referral and follow-up of the cases. SMA helped in the collection and interpretation of the data. EAA helped in the study deign and statistical analysis. All authors read and approved the final manuscript.

Funding

No funding sources

Availability of data and materials

Available with the corresponding author upon request

\section{Declarations}

Ethics approval and consent to participate

Written informed consent was signed by all patients before the examination. The study was approved by the ethics committee of the Faculty of Medicine, Cairo University. Reference number is not applicable. 


\section{Consent for publication}

All patients included in this research are above 16 years and all gave written informed consent to publish the data contained within this study.

\section{Competing interests}

The authors declare that they have no competing interests.

Received: 9 March 2021 Accepted: 13 May 2021

Published online: 26 May 2021

\section{References}

1. Priyanka J, Michelle S, Luciana PC, Myra F, Nicole H, Chenchan H, Aoife K, Sherelle LN, Refky N, Liina P, Anuradha SB, Angela T, Wendy V, Myles TT (2020) Endometriosis MRI lexicon: consensus statement from the Society of Abdominal Radiology Endometriosis Disease-Focused Panel. Abdominal Radiol 45(6):1552-1568

2. Foti PV, Farina R, Palmucci S, Vizzini IAA, Libertini N, Coronella M, Spadola S, Caltabiano R, Iraci M, Basile A, Milone P (2018) Endometriosis: clinical features, MR imaging findings and pathologic correlation. Insights into Imaging 9(2):149-172. https://doi.org/10.1007/s13244-017-0591-0

3. Corwin MT, Gerscovich EO, Lamba R, Wilson M, JP MG (2104) Differentiation of ovarian endometriomas from hemorrhagic cysts at MR imaging: utility of the T2 dark spot sign. Radiology 271(1):126-132

4. Thalluri AL, Knox S, Nguyen T (2017) MRI findings in deep infiltrating endometriosis: a pictorial essay. J Med Imaging Radiat Oncol 61(6):767-773. https://doi.org/10.1111/1754-9485.12680

5. Pin L, Monseau-Thiburce AC, Ziade-Coularis C, Benjamin A, Menut F, Brun $J$, Merlot B, Chateil JF (2019) Exploratory study of the interest of MR susceptibility-weighted imaging for the pre-operative assessment of pelvic endometriosis extent. Eur J Radiol 118:245-250. https://doi.org/10.1016/j.ejra d.2019.06.018

6. Lee NK, Kim S, Kim KH, Suh DS, Kim TU, Han GJ, Lee JW, Kim JY (2016) Diffusion-weighted magnetic resonance imaging in the differentiation of endometriomas from hemorrhagic cysts in the ovary. Acta Radiol 57(8):9981005. https://doi.org/10.1177/0284185115609805

7. Fernández RM, Ortega JB (2017) Magnetic resonance imaging of pelvic endometriosis. Radiología (English Edition) 59(4):286-296. https://doi.org/1 0.1016/j.rxeng.2017.06.002

8. Bazot M, Bharwani N, Huchon C, Kinkel K, Cunha TM, Guerra A, Manganaro L, Bunesch L, Kido A, Togashi K, Thomassin-Naggaral (2017) European Society of Urogenital Radiology (ESUR) guidelines: MR imaging of pelvic endometriosis. Eur Radiol 27(7):2765-2775. https://doi.org/10.1007/s00330016-4673-z

9. Nozomi T, Osamu Y, Eriko M, Shotaro N, Miyuki H, Kaori K, Osamu H, Masanobu N, Akashi T, Masaaki H, Shigeru S, Tomoyuki F, Yutaka O (2016) Usefulness of T2 star-weighted imaging in ovarian cysts and tumors. J Obstet Gynaecol Res 42(10):1336-1342

10. Carnahan M, Fedor J, Agarwal A, Gupta S (2013) Ovarian endometrioma: guidelines for selection of cases for surgical treatment or expectant management. Expert Rev Obstet Gynecol 8(1):29-55. https://doi.org/10.1 586/eog. 12.75

11. Woodward PJ, Sohaey R, Mezzetti TP Jr (2001) Endometriosis: radiologicpathologic correlation. Radiographics 21(1):193-216. https://doi.org/10.1148/ radiographics.21.1.g01ja14193

12. Outwater EK, Dunton CJ (1995) Imaging of the ovary and adnexa: clinical issues and applications of MR imaging. Radiology 194(1):1-18. https://doi. org/10.1148/radiology.194.1.7997533

13. Namimoto T, Yamashita Y, Awai K, Nakaura T, Yanaga Y, Hirai T, Saito T, Katabuchi H (2009) Combined use of T2-weighted and diffusion-weighted 3-T MR imaging for differentiating uterine sarcomas from benign leiomyomas. Eur Radiol 19(11):2756

14. Abd El-Dayem TM, Zaky YH, Al-Gizawy AM, Hassan HHM (2017) New magnetic resonance imaging sequences for the female pelvis in diagnosing ovarian endometrioma an imaging-laparoscopic correlation. J Gen Med Prim Care 1(1):1-8

15. Moteki T, Horikoshi H, Endo K (2002) Relationship between apparent diffusion coefficient and signal intensity in endometrial and other pelvic cysts. Magn Reson Imaging 20(6):463-470. https://doi.org/10.1016/S0730-72 5X(02)00524-6

16. Busard MPH, Mijatovic V, Van Kuijk C, Pieters-van den Bos IC, Hompes PGA, Van Waesberghe JHTM (2010) Magnetic resonance imaging in the evaluation of (deep infiltrating) endometriosis: the value of diffusionweighted imaging. J Magn Reson Imaging 31(5):1117-1123. https://doi. org/10.1002/jmri.22139

17. Takeuchi M, Matsuzaki K, Nishitani H (2008) Susceptibility-weighted MRI of endometrioma: preliminary results. Am J Roentgenol 191(5):1366-1370. https://doi.org/10.2214/AJR.07.3974

18. Takeuchi M, Matsuzaki K, Harada M (2015) Susceptibility-weighted MRI of extra-ovarian endometriosis: preliminary results. Abdom Imaging 40(7): 2512-2516. https://doi.org/10.1007/s00261-015-0378-z

\section{Publisher's Note}

Springer Nature remains neutral with regard to jurisdictional claims in published maps and institutional affiliations.

\section{Submit your manuscript to a SpringerOpen ${ }^{\circ}$ journal and benefit from:}

- Convenient online submission

- Rigorous peer review

- Open access: articles freely available online

- High visibility within the field

- Retaining the copyright to your article

Submit your next manuscript at $\boldsymbol{\nabla}$ springeropen.com 